\title{
Phase Behavior that Enables Solvent-Free Carbonate-Promoted Furoate Carboxylation
}

Amy D. Frankhouser and Matthew W. Kanan*

Department of Chemistry, Stanford University, Stanford, California 94305, United States

\begin{abstract}
Solvent-free chemistry has been used to streamline synthesis, reduce waste, and access novel reactivity, but the physical nature of the reaction medium in the absence of solvent is often poorly understood. Here we reveal the phase behavior that enables the solvent-free carboxylation reaction in which carbonate, furan-2-carboxylate (furoate), and $\mathrm{CO}_{2}$ react to form furan-2,5-dicarboxylate $\left(\mathrm{FDCA}^{2-}\right)$. This transformation has no solution-phase analog and can be applied to convert lignocellulose into performance-advantaged plastics. Using operando powder X-ray diffraction and thermal analysis to elucidate the temperature- and conversion-dependent phase composition, we find that the reaction medium is a heterogeneous mixture of a ternary eutectic molten phase, solid $\mathrm{Cs}_{2} \mathrm{CO}_{3}$, and solid $\mathrm{Cs}_{2} \mathrm{FDCA}$. During the reaction, the molten phase and solid $\mathrm{Cs}_{2} \mathrm{CO}_{3}$ diminish as solid $\mathrm{Cs}_{2} \mathrm{FDCA}$ accumulates. These insights are critical for increasing the scale of furoate carboxylation and provide a framework to guide the development of other solvent-free transformations.
\end{abstract}

\section{Main Text}

Solvent-free reactions have been investigated primarily as a means to improve the practicality of conventional solution-phase reactions by reducing waste, accelerating the rate, or simplifying purification. ${ }^{1-9}$ The unusual properties of a reaction medium composed of neat reactants also raise the possibility of accessing reactivity that is very difficult or impossible to recapitulate in solution. To this end, we recently showed that carbonate $\left(\mathrm{CO}_{3}{ }^{2-}\right)$ can promote $\mathrm{C}-\mathrm{H}$ carboxylation of very weakly acidic aryl $\mathrm{C}-\mathrm{H}$ bonds $\left(\mathrm{p} K_{\mathrm{a}}>35\right.$ in organic solvent) in carefully selected solvent-free alkali salts at elevated temperature. ${ }^{10-13}$ By contrast, even under forcing conditions in organic solvent, $\mathrm{CO}_{3}{ }^{2-}$ has been found to be incapable of promoting carboxylation of $\mathrm{C}-\mathrm{H}$ bonds with $\mathrm{p} K_{\mathrm{a}}>27 .{ }^{14}$ The ability to use a simple, regenerable base such as $\mathrm{CO}_{3}{ }^{2-}$ is essential if carboxylation is to be applied to scalable chemical synthesis. Solvent-free alkali salts unlock this possibility for some targets, but a better understanding of the reaction medium is needed to realize this potential. 


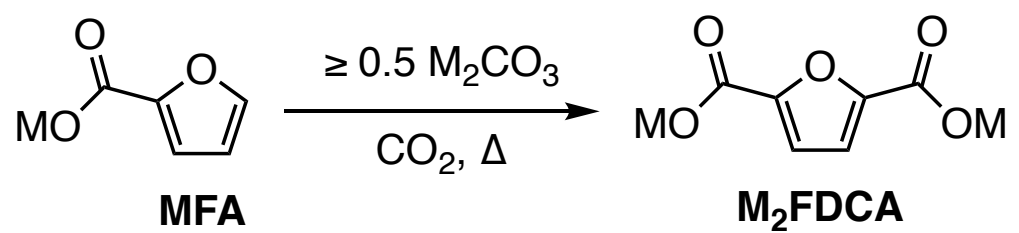

\begin{tabular}{|cc|}
\hline $\begin{array}{c}\mathrm{M}=\mathrm{Cs} \\
\mathrm{M}=\mathrm{K}\end{array}$ & Up to $89 \%$ Yield \\
No Reaction
\end{tabular}

Scheme 1 | Solvent-free carbonate-promoted furoate carboxylation

A promising application of solvent-free $\mathrm{CO}_{3}{ }^{2-}$-promoted $\mathrm{C}-\mathrm{H}$ carboxylation is the conversion of furan-2-carboxylate (furoate) into furan-2,5-dicarboxylate $\left(\mathrm{FDCA}^{2-}\right)$ (Scheme 1). The protonated form of the carboxylation product - furan-2,5-dicarboxylic acid (FDCA) - is a potential platform chemical for the synthesis of performance-advantaged plastics. ${ }^{15,16}$ Extensive efforts to produce FDCA on a commercial scale from $\mathrm{C}_{6}$ sugars (glucose and fructose) have been impeded by the technical difficulties and process complexity of dehydrating and oxidizing $\mathrm{C}_{6}$ sugars. ${ }^{17} \mathrm{C}-\mathrm{H}$ carboxylation opens a new route to FDCA from underutilized $\mathrm{C}_{5}$ sugar feedstocks (inedible hemicellulose) and avoids many of the process challenges that have plagued $\mathrm{C}_{6}$ routes. ${ }^{11}$

In its simplest form, furoate carboxylation is performed by combining alkali furoate (MFA) with at least 0.5 equivalents of alkali carbonate and heating the salt mixture under $\mathrm{CO}_{2}$. Yields of FDCA $^{2-}$ up to $89 \%$ have been obtained in laboratory-scale experiments (up to $>100 \mathrm{~g}$ ), but the performance shows a strong dependence on the identity of the alkali cation (Scheme 1). When using a single alkali cation, high yields of $\mathrm{FDCA}^{2-}$ can be achieved with $\mathrm{Cs}^{+}$, but no detectable yield is obtained with any of the other alkali cations. Herein, we present a detailed investigation of the physical and structural properties of the reaction mixture components for solvent-free furoate carboxylation. Using a combination of X-ray diffraction and thermal analysis techniques, we elucidate the phase behavior that enables solvent-free furoate carboxylation in $\mathrm{Cs}^{+}$salts and explain the lack of reactivity in pure $\mathrm{K}^{+}$salts. Our findings reveal how reactivity in this system depends on the phase equilibria that exist within multi-component salt mixtures.

In the solvent-free furoate carboxylation reaction, the material that makes up the reaction medium consists initially of a mixture of alkali furoate (MFA) and alkali carbonate $\left(\mathrm{M}_{2} \mathrm{CO}_{3}\right)$. Alkali carbonates are inorganic crystalline solids with high melting points $\left(\mathrm{T}_{\mathrm{m}}=899^{\circ} \mathrm{C}\right.$ for $\mathrm{M}=\mathrm{K} ; \mathrm{T}_{\mathrm{m}}=$ 
$793{ }^{\circ} \mathrm{C}$ for $\left.\mathrm{M}=\mathrm{Cs}\right) \cdot{ }^{18}$ Hence, any phase behavior that occurs in the mixture at carboxylation temperatures $\left(<280{ }^{\circ} \mathrm{C}\right)$ can be expected to derive primarily from the furoate component. To understand the cation dependence of furoate carboxylation reactivity, we looked first to contrast the structural and thermal properties of potassium and cesium furoate.

Both potassium furoate (KFA) and cesium furoate (CsFA) are crystalline solids at room temperature, though $\mathrm{CsFA}$ is distinctly more hygroscopic. A single crystal X-ray structure obtained for KFA shows that the salt packs in the form of 2-dimensional sheets of potassium cations sandwiched between organic anion layers (Figs. 1a, S1, S2). In contrast to KFA, diffraction-quality single crystals of CsFA could not be isolated for determination of a crystal structure, a common problem for aromatic carboxylates. ${ }^{19}$ However, ambient temperature synchrotron Powder X-ray Diffraction (PXRD) patterns of anhydrous CsFA indicate that the salt also possesses a layered structure, evidenced by an intense low-index peak. A plausible room temperature structure for CsFA was determined from PXRD data by a simulated annealing procedure ${ }^{20}$ after indexing of ambient temperature PXRD patterns for anhydrous CsFA collected at two independent synchrotron beamlines (Figs. S3-S5, see Supporting Information for additional details). The packing in the resulting structure (Fig. 1b) is very similar to that in KFA.

Given the similarity of potassium and cesium furoate at ambient temperature, any differences in the properties of the two salts that are related to their disparity in carboxylation reactivity must manifest only at elevated temperatures. Thermal analysis reveals that the key difference between KFA and CsFA is that only the latter salt can exist as a stable isotropic melt. As with many other alkali carboxylate salts, ${ }^{21-23}$ alkali furoates were found to exhibit complex polymorphism. Differential Scanning Calorimetry (DSC) thermograms collected for samples of KFA and CsFA show that upon heating from room temperature, both salts undergo a series of endothermic events prior to the onset of exothermic decomposition (Fig. 1c,d). In both cases, the highest temperature endothermic transition was identified as melting $\left(\mathrm{T}_{\mathrm{m}}=390{ }^{\circ} \mathrm{C}\right.$ for $\mathrm{M}=\mathrm{K} ; \mathrm{T}_{\mathrm{m}}=264{ }^{\circ} \mathrm{C}$ for $\mathrm{M}=$ $\mathrm{Cs}$ ), while the lower temperature endothermic events correspond to solid-solid transitions between crystalline or semi-crystalline MFA polymorphs (denoted by Roman numerals increasing with temperature from I for the ambient temperature polymorph). The assignment of these thermal events was further corroborated by Thermogravimetric Analysis, Polarized Optical Microscopy, and Variable Temperature PXRD techniques (Figs. S6-S11). Importantly, while the melting 
a

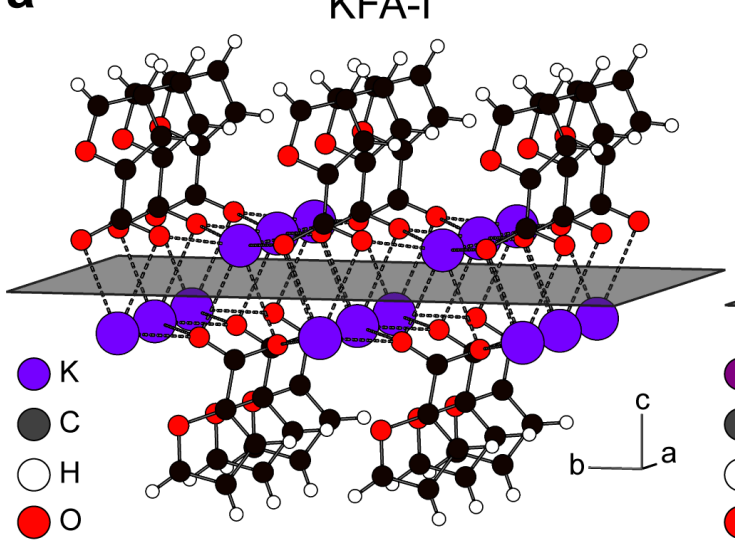

b
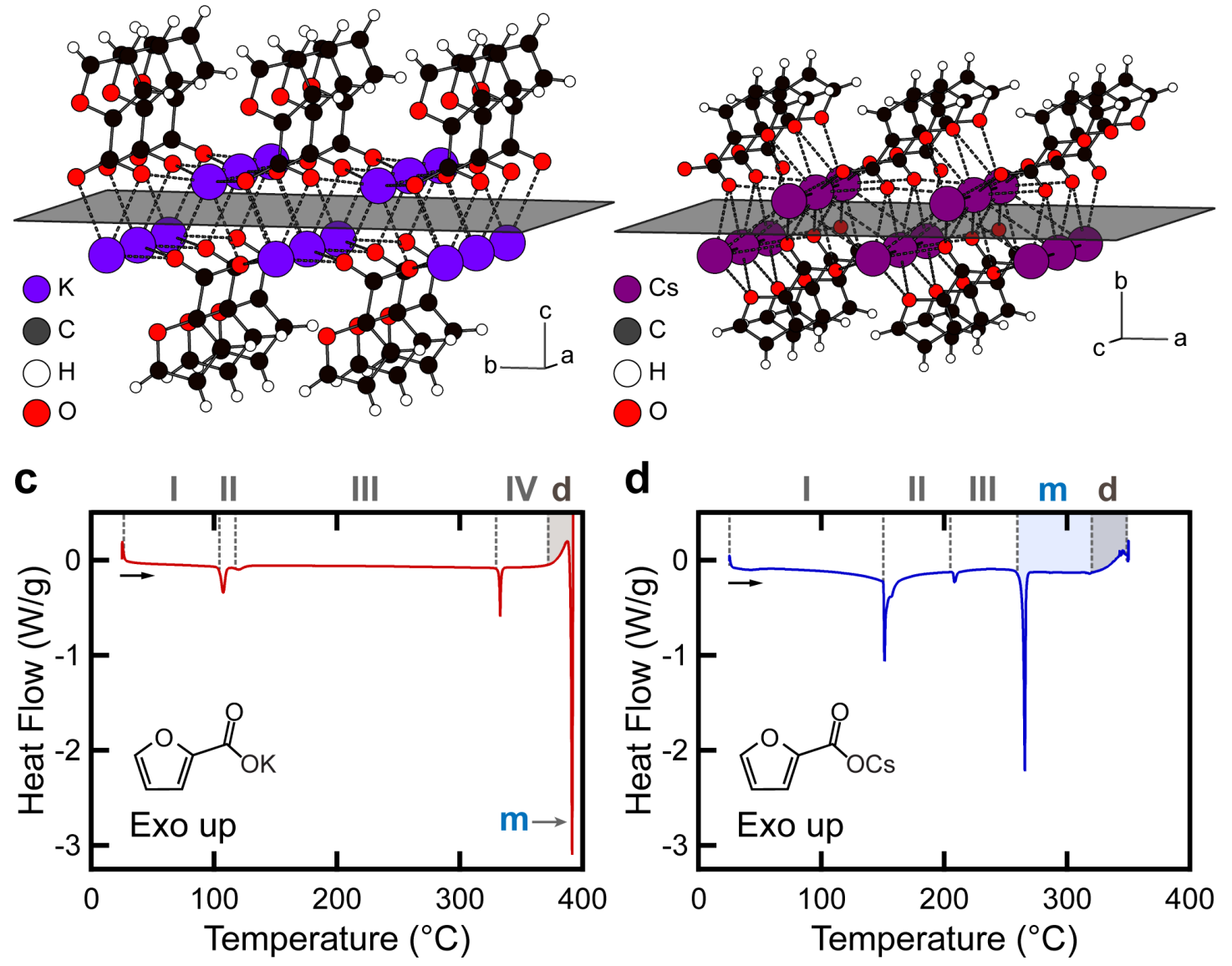

Figure 1 | Structural and thermal properties of alkali furoate salts. a, Layered structure of the ambient temperature polymorph of potassium furoate (KFA), as determined from refined single crystal X-ray diffraction data. The (001)-family plane bisecting the cation layer is shown for clarity. b, Layered structure of the ambient temperature polymorph of cesium furoate (CsFA), as determined from synchrotron powder X-ray diffraction data by simulated annealing. The (010)-family plane bisecting the cation layer is shown for clarity. c,d, Differential Scanning Calorimetry heating curves of KFA (c) and CsFA (d) salts. Temperature regions corresponding to different crystalline polymorphs are labeled above the plots. The region marked "d" corresponds to thermal decomposition, while the peak (or region) marked "m" corresponds to the isotropic melt.

transition for KFA overlaps with its region of thermal decomposition, melting of CsFA occurs approximately $65^{\circ} \mathrm{C}$ below the onset of its own decomposition event $\left(\mathrm{T}_{\mathrm{d}}=328^{\circ} \mathrm{C}\right)$. The ability of CsFA to exist in a molten form is crucial for its ability to participate in solvent-free chemistry.

The furoate salt is only one component of the carboxylation reaction medium, however. The stoichiometry of furoate carboxylation promoted by $\mathrm{M}_{2} \mathrm{CO}_{3}$ requires a minimum of 0.5 molar equivalents of $\mathrm{M}_{2} \mathrm{CO}_{3}$ relative to $\mathrm{MFA}$ in order to achieve complete conversion to the alkali dicarboxylate salt $\left(\mathrm{M}_{2} \mathrm{FDCA}\right)$. In order to understand the physical properties of the reaction 
mixture at the outset of a solvent-free furoate carboxylation reaction, the impact of the addition of $\mathrm{M}_{2} \mathrm{CO}_{3}$ on the thermal properties of the MFA salt must be examined.

A combination of thermal analysis and variable temperature PXRD reveals that $\mathrm{CsFA}$ and $\mathrm{Cs}_{2} \mathrm{CO}_{3}$ constitute a binary eutectic system (Fig. 2a). DSC thermograms were collected for a series of mixtures of $\mathrm{CsFA}$ and $\mathrm{Cs}_{2} \mathrm{CO}_{3}$ salts with varying composition. Increasing the $\mathrm{Cs}_{2} \mathrm{CO}_{3}$ mole fraction ( $\mathrm{x}$ ) in the mixture affects the position and appearance of the final endothermic transition observed in the DSC heating curve (Fig. 2b). When a very small amount of $\mathrm{Cs}_{2} \mathrm{CO}_{3}$ is present ( $\mathrm{x}$ $=0.01$ ), the transition shifts to a lower temperature relative to the melting transition observed for pure CsFA. The peak also presents with a distinct shoulder, with the onset of this shoulder occurring at $\mathrm{T}=256^{\circ} \mathrm{C}$. For higher values of $\mathrm{x}$, only a single endothermic event is observed, also with an onset temperature of approximately $256{ }^{\circ} \mathrm{C}$. Variable temperature PXRD was used to further examine the nature of this transition, using $\mathrm{x}=0.35$ to match the $1: 0.55 \mathrm{CsFA}: \mathrm{Cs}_{2} \mathrm{CO}_{3}$ stoichiometry most commonly employed in the synthetic reaction. ${ }^{10}$ Comparison of PXRD patterns collected before and after heating this mixture of $\mathrm{CsFA}$ and $\mathrm{Cs}_{2} \mathrm{CO}_{3}$ past $256{ }^{\circ} \mathrm{C}$ shows that the transition corresponds to the selective disappearance of diffraction peaks attributable to solid CsFA-III, while anhydrous crystalline $\mathrm{Cs}_{2} \mathrm{CO}_{3}$ remains (Fig. 2c). Moreover, the transition was shown to be reversible. Immediately cooling the mixture upon disappearance of the CsFA peaks leads to their reappearance as CsFA-III recrystallizes at approximately the same temperature (Fig. 2d).

These observations are all consistent with a lowering of the CsFA melting point by $\sim{ }^{\circ} \mathrm{C}$ upon addition of $\mathrm{Cs}_{2} \mathrm{CO}_{3}$ through formation of a eutectic. For all compositions of relevance to the CsFA carboxylation reaction (i.e. $x \geq 0.33$ ), the reaction necessarily occurs from a heterogeneous mixture of solid crystalline $\mathrm{Cs}_{2} \mathrm{CO}_{3}$ and a molten eutectic which contains only a small fraction of the $\mathrm{Cs}_{2} \mathrm{CO}_{3}$ base. It should be noted that although a two-phase region of coexistence between solid $\mathrm{Cs}_{2} \mathrm{CO}_{3}$ and molten eutectic is present, the liquidus temperature at which the mixture would theoretically become uniformly molten can never be reached because it lies well beyond the thermal decomposition temperature of CsFA.

In contrast to the $\mathrm{CsFA}-\mathrm{Cs}_{2} \mathrm{CO}_{3}$ system, the analogous mixture with $\mathrm{M}=\mathrm{K}$ does not exhibit a stable eutectic melt. The absence of eutectic melting was demonstrated using variable temperature 

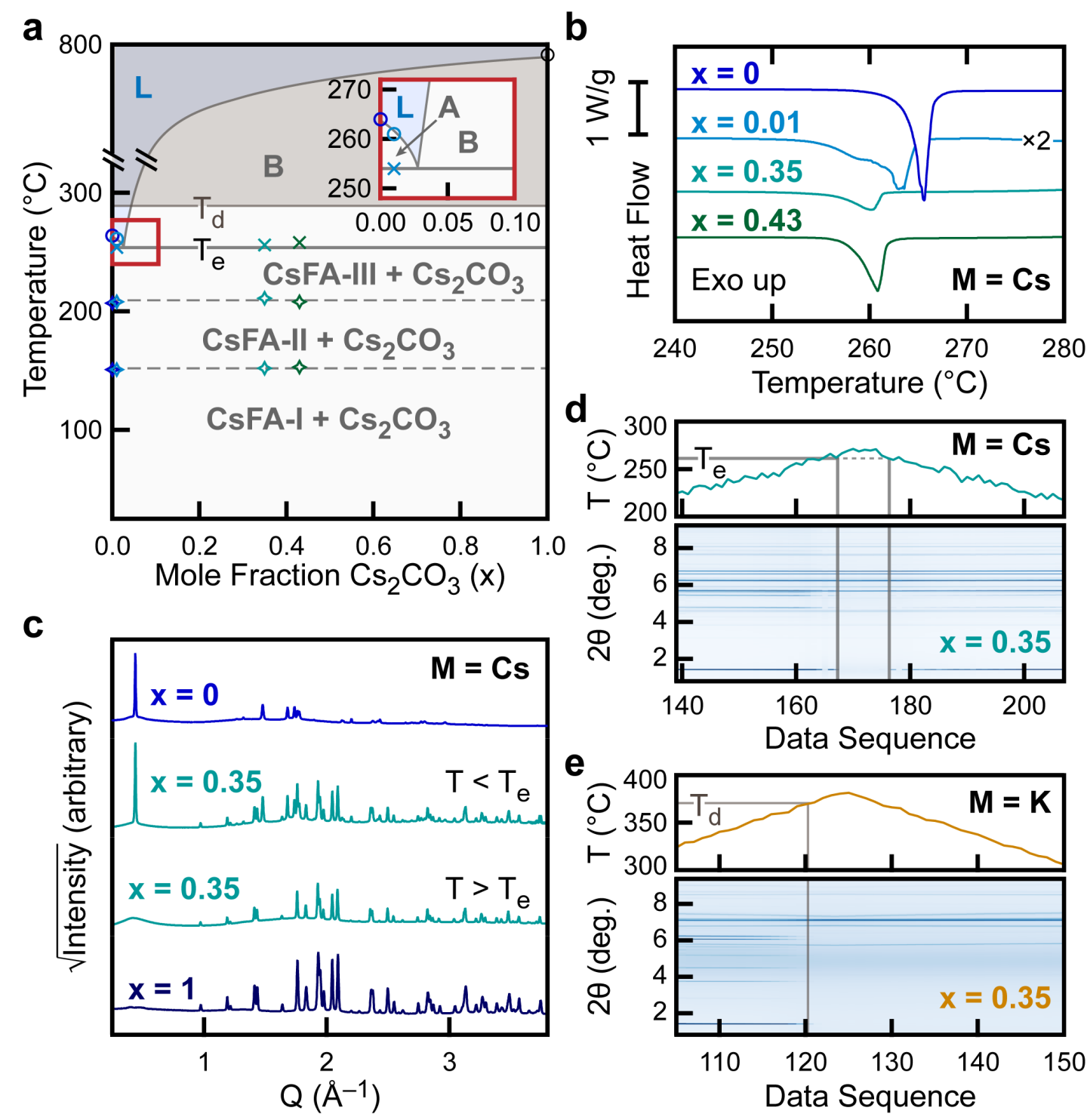

Figure $2 \mid$ Phase equilibria in binary $\mathrm{MFA}-\mathrm{M}_{2} \mathrm{CO}_{3}$ mixtures. a, Phase equilibrium diagram for the anhydrous system $\mathrm{CsFA}-\mathrm{Cs}_{2} \mathrm{CO}_{3}$. Marked temperatures of melting transitions ( $(\circ)$, eutectic transitions $(\times)$, and solid-solid phase transitions $(\diamond)$ were determined from DSC measurements. All two-phase regions below the eutectic temperature are labeled with their constituent solid phases. The region $\mathrm{L}$ corresponds to the uniform melt. The region A (inset) corresponds to the two-phase mixture of CsFA-III + eutectic melt and $\mathrm{B}$ corresponds to the two-phase mixture of $\mathrm{Cs}_{2} \mathrm{CO}_{3}+$ eutectic melt. All regions above the line marked $T_{d}$ are inaccessible due to decomposition of the CsFA component. b, DSC thermograms comparing melting of pure $\mathrm{CsFA}$ with eutectic melting in $\mathrm{CsFA}-\mathrm{Cs}_{2} \mathrm{CO}_{3}$ mixtures of varying composition. The trace for $\mathrm{x}=0.01$ has been scaled vertically to highlight the two distinct thermal events (eutectic formation and liquidus melting) observed. c, PXRD patterns comparing a mixture of CsFA and $\mathrm{Cs}_{2} \mathrm{CO}_{3}$ with $\mathrm{x}=0.35$ before and after its eutectic melting event. Shown for reference are powder patterns for pure CsFA-III $(\mathrm{x}=0)$ and pure $\mathrm{Cs}_{2} \mathrm{CO}_{3}(\mathrm{x}=1)$. $\mathbf{d}, \mathbf{e}$ Temperature profile (top) and PXRD contour plot (bottom) for variable temperature PXRD $(\lambda=0.35380,5 \mathrm{sccm}$ He gas) performed on a mixture of CsFA and $\mathrm{Cs}_{2} \mathrm{CO}_{3}$ with $\mathrm{x}=0.35$ (d) or KFA and $\mathrm{K}_{2} \mathrm{CO}_{3}$ with $\mathrm{x}=0.35$ (e). Diffraction intensity in the contour plots increases from white to blue. Melting or decomposition events as evidenced by the disappearance of diffraction peaks are marked. 
PXRD. When a mixture of KFA and $\mathrm{K}_{2} \mathrm{CO}_{3}$ with $\mathrm{x}=0.35$ was heated, the peaks corresponding to the furoate component eventually disappeared, while the carbonate peaks remained. However, unlike in the cesium-containing mixture, in this case the peaks corresponding to the furoate component did not reappear upon immediate cooling, indicating KFA decomposition rather than a reversible eutectic melting event (Fig. 2e). In other words, the addition of $\mathrm{K}_{2} \mathrm{CO}_{3}$ to KFA does not sufficiently depress the KFA melting point to create a stable two-phase region containing molten KFA below its decomposition temperature.

At elevated temperature and in the presence of $\mathrm{CO}_{2}$ gas, a mixture of $\mathrm{CsFA}$ and $\mathrm{Cs}_{2} \mathrm{CO}_{3}$ will begin to convert to $\mathrm{Cs}_{2} \mathrm{FDCA}$. If the reaction is performed under flowing conditions, the water produced as a byproduct of carboxylation is stripped away, and the carboxylation process can be envisioned as an interaction between gaseous $\mathrm{CO}_{2}$ and a three-component mixture of anhydrous salts: CsFA, $\mathrm{Cs}_{2} \mathrm{CO}_{3}$, and $\mathrm{Cs}_{2} \mathrm{FDCA}$. In an ideal scenario where the reaction proceeds with $100 \%$ selectivity to form exclusively $\mathrm{Cs}_{2} \mathrm{FDCA}$, the progression of the reaction from starting material to full conversion can be represented as a traversal through ternary phase space ${ }^{24,25}$ with a compositional constraint dictated by the 2:1 stoichiometric relationship between CsFA and $\mathrm{Cs}_{2} \mathrm{CO}_{3}$ (Fig. 3a). We examined the phase equilibrium of this ternary system by performing thermal and PXRD analysis on samples of $\mathrm{CsFA}-\mathrm{Cs}_{2} \mathrm{CO}_{3}-\mathrm{Cs}_{2} \mathrm{FDCA}$ mixtures with compositions designed to mimic the progression of a reaction starting from a binary $\mathrm{CsFA}-\mathrm{Cs}_{2} \mathrm{CO}_{3}$ mixture with $\mathrm{x}=0.35$.

Although $\mathrm{Cs}_{2} \mathrm{FDCA}$ by itself is a crystalline solid in the temperature range relevant for CsFA carboxylation (Figs. S14 and S15), conversion of the starting materials to $\mathrm{Cs}_{2} \mathrm{FDCA}$ substantially alters the thermal transitions of the mixture compared to the $\mathrm{CsFA}-\mathrm{Cs}_{2} \mathrm{CO}_{3}$ binary phase system. DSC thermograms of anhydrous ternary mixtures prepared to mimic varying extents of conversion in the carboxylation reaction (defined here as $\mathrm{Y}, 0 \leq \mathrm{Y} \leq 1$ ) show that the accumulation of even small amounts of $\mathrm{Cs}_{2} \mathrm{FDCA}$ leads to depression of the system's eutectic temperature $\mathrm{T}_{\mathrm{e}}$ below its value in the binary system (Fig. 3b). With $25 \%$ of the starting CsFA converted to $\mathrm{Cs}_{2} \mathrm{FDCA}$, the mixture's eutectic temperature was lowered to $213{ }^{\circ} \mathrm{C}$, which is $\sim 43{ }^{\circ} \mathrm{C}$ below the $\mathrm{CsFA}-\mathrm{Cs}_{2} \mathrm{CO}_{3}$ binary eutectic melting point and within a few degrees of the transition between forms II and III of pure CsFA. Comparison of PXRD patterns taken before and after the eutectic transition in ternary mixtures with $\mathrm{Y}=0.05$ and 0.25 illustrates which crystalline phases are present in the mixture before and after eutectic melting (Fig. 3c-e). This comparison affirms that eutectic melting 
a

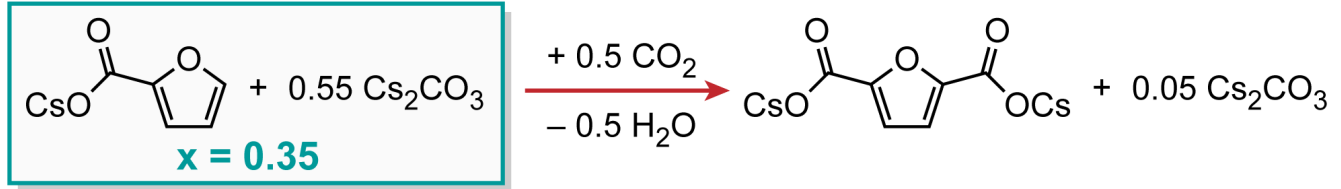

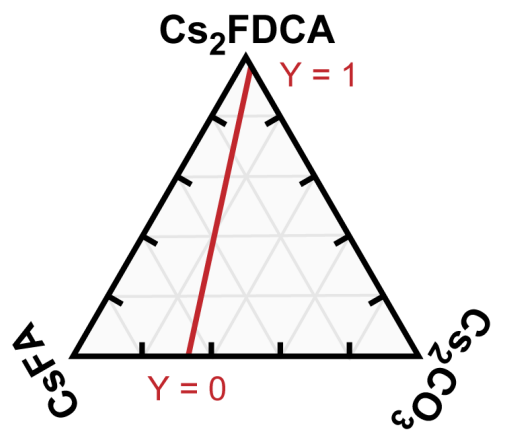
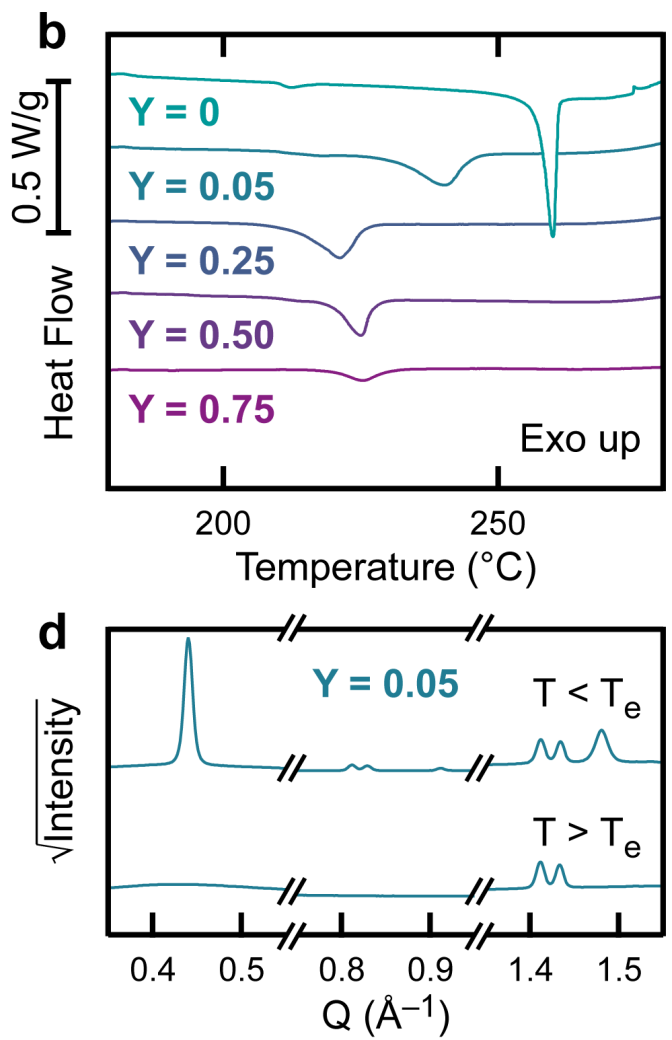
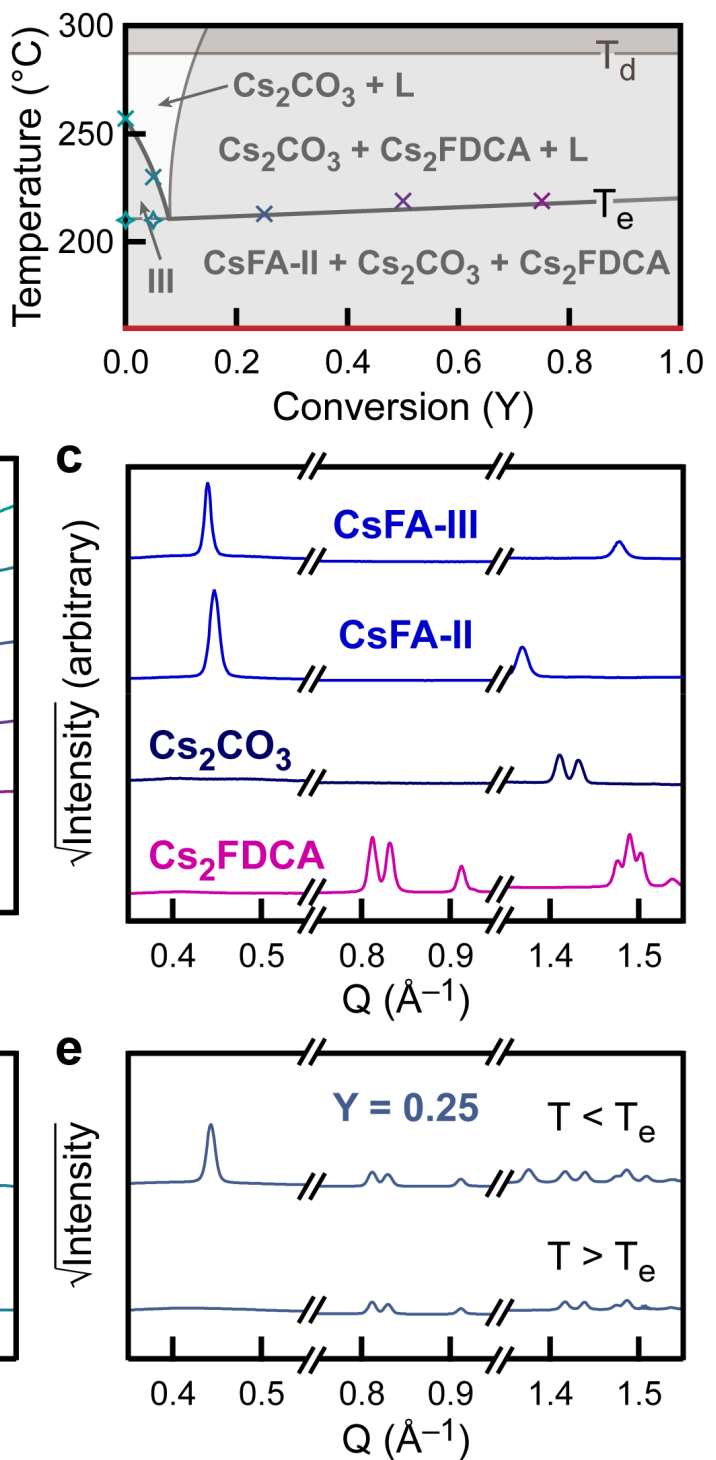

Figure 3 | Phase equilibrium as a function of conversion in a ternary $\mathrm{CsFA}_{4}-\mathrm{Cs}_{2} \mathrm{CO}_{3}-\mathrm{Cs}_{2} \mathrm{FDCA}$ mixture. a, Schematic representation of the cesium furoate carboxylation reaction as a cross-section of ternary phase space. Regions in the phase equilibrium diagram (lower right) are labeled with their constituent phases, where "L" corresponds to the molten eutectic phase. The region "III" corresponds to the three-phase region consisting of solid CsFA-III $+\mathrm{Cs}_{2} \mathrm{CO}_{3}+\mathrm{Cs}_{2} \mathrm{FDCA}$. b, DSC thermograms comparing eutectic melting transitions in the ternary mixture as a function of conversion. c, Elevated temperature PXRD patterns of individual components of the ternary mixture. Selected regions of the patterns are shown that allow for identification of the constituent crystalline phases in patterns of multicomponent mixtures. Patterns shown for $\mathrm{Cs}_{2} \mathrm{CO}_{3}$ and $\mathrm{Cs}_{2} \mathrm{FDCA}$ are for their anhydrous, high temperature forms. d,e, PXRD patterns comparing ternary mixtures with $\mathrm{Y}=0.05$ (d) and $\mathrm{Y}=0.25$ (e) before and after eutectic melting, demonstrating that $\mathrm{Cs}_{2}$ FDCA is fully dissolved in the melt only at low conversion. 
of the CsFA component at higher values of Y occurs directly from CsFA-II, bypassing the CsFAIII polymorph. An additional observation that emerges from this PXRD analysis is that a distinct region exists at low conversions for which the $\mathrm{Cs}_{2}$ FDCA salt is fully soluble in the eutectic melt. Eutectic melting in a mixture with $\mathrm{Y}=0.05$ presents as the disappearance of diffraction peaks attributable to both the CsFA-III and $\mathrm{Cs}_{2}$ FDCA components (Fig. 3d). For a mixture with $\mathrm{Y}=$ 0.25 , however, $\mathrm{Cs}_{2} \mathrm{FDCA}$ diffraction peaks remain even after the disappearance of crystalline CsFA-II (Fig. 3e). While increasing conversion leads to lowering of the eutectic melting temperature, the molten eutectic fraction diminishes as the mixture becomes increasingly rich in $\mathrm{Cs}_{2}$ FDCA. This effect can be seen from the decreasing peak area of the endothermic event associated with eutectic melting (Fig. 3b). Otherwise put, the carboxylation of CsFA occurs in a mixture that changes its phase composition as a function of conversion. The unique reaction medium is initially a heterogeneous blend of solid and molten material that becomes progressively less molten in character as full conversion is approached.

Finally, we looked to directly observe the carboxylation of CsFA using operando PXRD. The results of these studies are consistent with the ternary phase equilibrium model based on the above analysis of ternary mixtures that mimic different extents of conversion, and additionally demonstrate some interesting properties of CsFA as a solvent-free reactant. In two separate experiments, mixtures of $\mathrm{CsFA}$ and $\mathrm{Cs}_{2} \mathrm{CO}_{3}$ with $\mathrm{x}=0.35$ were heated while flowing $\mathrm{CO}_{2}$ through the sample capillary at $5 \mathrm{sccm}$. Following an initial ramp up to the chosen reaction temperature, each sample was held at its setpoint for $70 \mathrm{~min}$ while diffraction images were collected continuously. The two chosen setpoints were 260 and $250{ }^{\circ} \mathrm{C}$ - above and below the eutectic temperature of the CsFA- $\mathrm{Cs}_{2} \mathrm{CO}_{3}$ binary mixture, respectively (Fig. 4a). As expected, when the reaction was conducted at $260{ }^{\circ} \mathrm{C}$, eutectic melting occurred during the temperature ramp, just prior to reaching $260{ }^{\circ} \mathrm{C}$. As the reaction proceeded and conversion increased, no significant changes were initially observed in the sample's PXRD pattern. However, peaks corresponding to crystalline $\mathrm{Cs}_{2} \mathrm{FDCA}$ became visible after approximately $30 \mathrm{~min}$ at the reaction temperature had elapsed (Fig. 4b). Thus, starting from the binary eutectic melt, carboxylation forms FDCA ${ }^{2-}$ that is initially dissolved in the melt. As conversion increases, a saturation point is reached (somewhere between $\mathrm{Y}=0.05$ and $\mathrm{Y}=0.25$ ) and crystalline $\mathrm{Cs}_{2} \mathrm{FDCA}$ forms as an additional phase. 

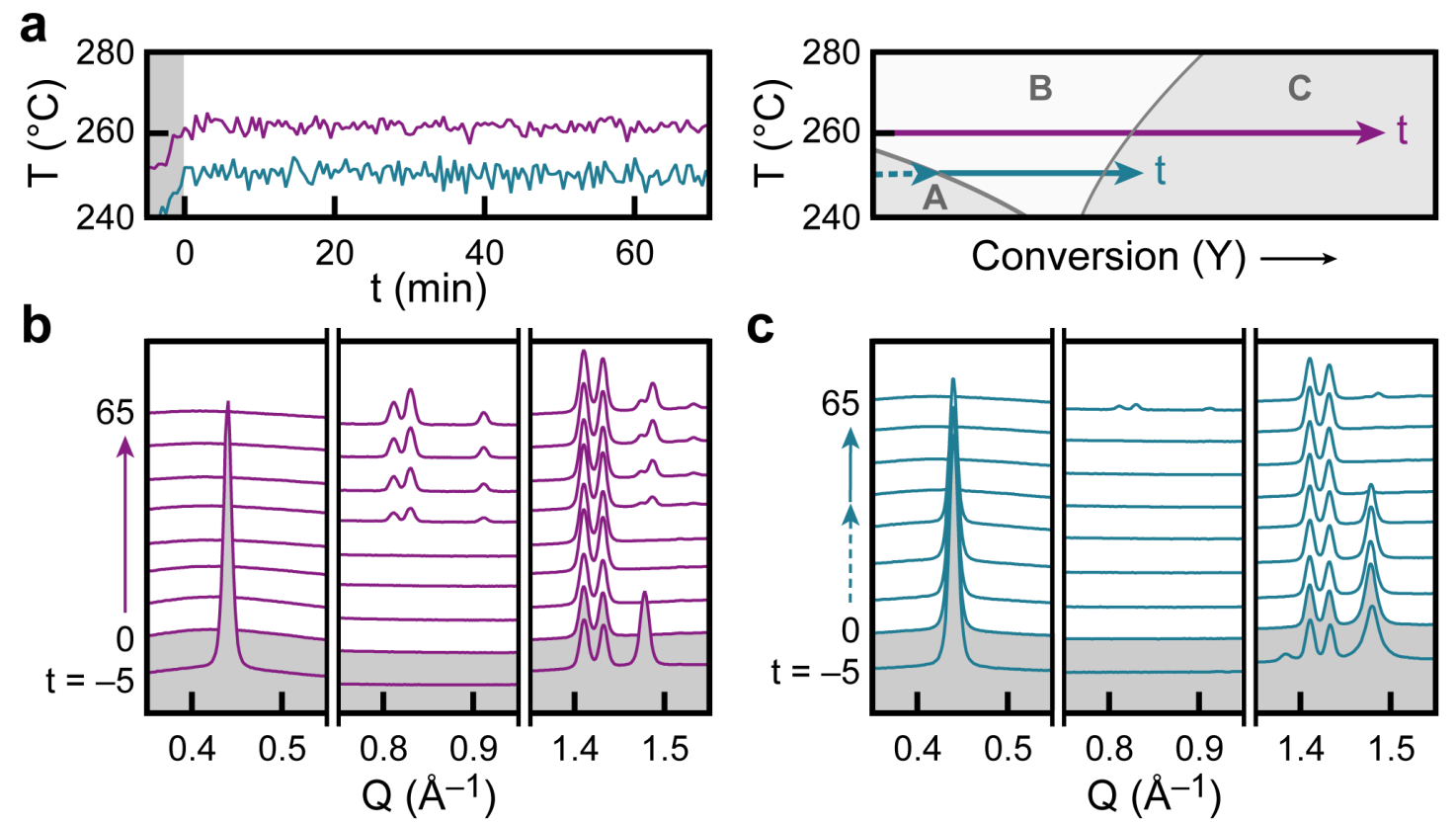

Figure 4 | Operando PXRD analysis of cesium furoate carboxylation. a, Measured temperature profiles of two operando PXRD experiments (left) and representation of ternary phase regions traversed at each temperature (right). Both experiments were performed by heating mixtures of $\mathrm{CsFA}$ and $\mathrm{Cs}_{2} \mathrm{CO}_{3}(\mathrm{x}=$ 0.35) while flowing $\mathrm{CO}_{2}$ gas through the sample capillary at $5 \mathrm{sccm}$ and collecting diffraction images at $30 \mathrm{~s}$ intervals. For each experiment, the time $t=0$ is defined as the point at which the setpoint temperature was first reached. Phase regions in the diagram correspond to CsFA-III $+\mathrm{Cs}_{2} \mathrm{CO}_{3}+\mathrm{Cs}_{2} \mathrm{FDCA}$ (A), $\mathrm{Cs}_{2} \mathrm{CO}_{3}+$ eutectic melt (B), and $\mathrm{Cs}_{2} \mathrm{CO}_{3}+\mathrm{Cs}_{2} \mathrm{FDCA}+$ eutectic melt (C). b,c, Selected PXRD patterns showing the evolution of crystalline phases for reactions carried out with setpoint temperatures of $260{ }^{\circ} \mathrm{C}$ (b) and $250^{\circ} \mathrm{C}(\mathbf{c})$.

When a fresh sample was heated instead to $250{ }^{\circ} \mathrm{C}$ under flowing $\mathrm{CO}_{2}$, no melting event was initially observed. We anticipated that no $\mathrm{Cs}_{2} \mathrm{FDCA}$ formation would occur in the absence of this initial eutectic melting. Surprisingly, after approximately $35 \mathrm{~min}$ at $250{ }^{\circ} \mathrm{C}$, spontaneous melting of the CsFA component was observed. As no appreciable change to the sample's temperature occurred during this interval, the occurrence of this melting event must have been induced instead by a change in the mixture composition. At the conclusion of the $70 \mathrm{~min}$ reaction period, peaks corresponding to crystalline $\mathrm{Cs}_{2}$ FDCA became barely visible in the PXRD pattern, confirming that carboxylation occurred over the course of the experiment (Fig. 4c). This result highlights the melting temperature depression induced by even small amounts of $\mathrm{Cs}_{2} \mathrm{FDCA}$. In addition, it suggests that a small degree of reactivity is possible between CsFA-III and $\mathrm{Cs}_{2} \mathrm{CO}_{3}$ even without the formation of bulk eutectic domains between the two salts (see Discussion). Relative to lowertemperature polymorphs of CsFA and the solid forms of KFA, CsFA-III shows only weak 
crystalline ordering (Figs. S9, S10), which may be sufficient to enable CsFA to participate in intermolecular reactions.

Integrating the results above, Figure 5 depicts the evolution of the phase composition for an idealized CsFA carboxylation reaction performed at a temperature just beyond the binary eutectic melting temperature. Upon reaching the eutectic melting temperature, the initial mixture of solid CsFA and $\mathrm{Cs}_{2} \mathrm{CO}_{3}$ transitions into a mixture of solid and molten components. The molten phase is initially a binary eutectic composed primarily of the low-melting CsFA with a minor amount of $\mathrm{Cs}_{2} \mathrm{CO}_{3}$; most of the $\mathrm{Cs}_{2} \mathrm{CO}_{3}$ remains in its solid, crystalline form. As the reaction begins and $\mathrm{Cs}_{2} \mathrm{FDCA}$ is formed, it is initially dissolved in the molten phase. With increased conversion, $\mathrm{Cs}_{2} \mathrm{FDCA}$ saturates the molten phase and precipitates to form crystalline $\mathrm{Cs}_{2} \mathrm{FDCA}$ as an additional solid phase. The reaction proceeds in the ternary eutectic phase with the minor eutectic components $\left(\mathrm{Cs}_{2} \mathrm{CO}_{3}\right.$ and $\left.\mathrm{Cs}_{2} \mathrm{FDCA}\right)$ in equilibrium with their corresponding solid phases. Increasing conversion leads to the gradual consumption of the ternary eutectic and solid $\mathrm{Cs}_{2} \mathrm{CO}_{3}$ components of the mixture as the solid $\mathrm{Cs}_{2}$ FDCA increases.

The idealized reaction presented in Figure 5 does not account for the small to moderate amounts of decomposition products that are typically observed along with the target $\mathrm{Cs}_{2}$ FDCA product, which complicate the reaction mixture composition. ${ }^{10,11}$ Some of these decomposition products are small-molecule carboxylate salts (e.g. formate, acetate, malonate) that are comparatively lowmelting and might alter the phase behavior of the molten component of the reaction mixture. However, the most significant byproduct observed is dense, polymeric char that adds an additional solid component to the heterogeneous mixture. Notwithstanding these additional complexities, the components investigated in the experiments above make up the majority of the material present in the CsFA carboxylation reaction.

The observation of CsFA carboxylation for a reaction performed below the binary eutectic melting temperature (Fig. 4c) suggests that it is possible for intermolecular reactions to occur between two solid phases (in this case CsFA-III and $\mathrm{Cs}_{2} \mathrm{CO}_{3}$ ). Carboxylation of CsFA initially proceeds in this ostensibly all-solid mixture until formation of a very small amount of the product $\mathrm{Cs}_{2} \mathrm{FDCA}$ induces melting as a ternary eutectic. Evidence for intermolecular reactions between species in 

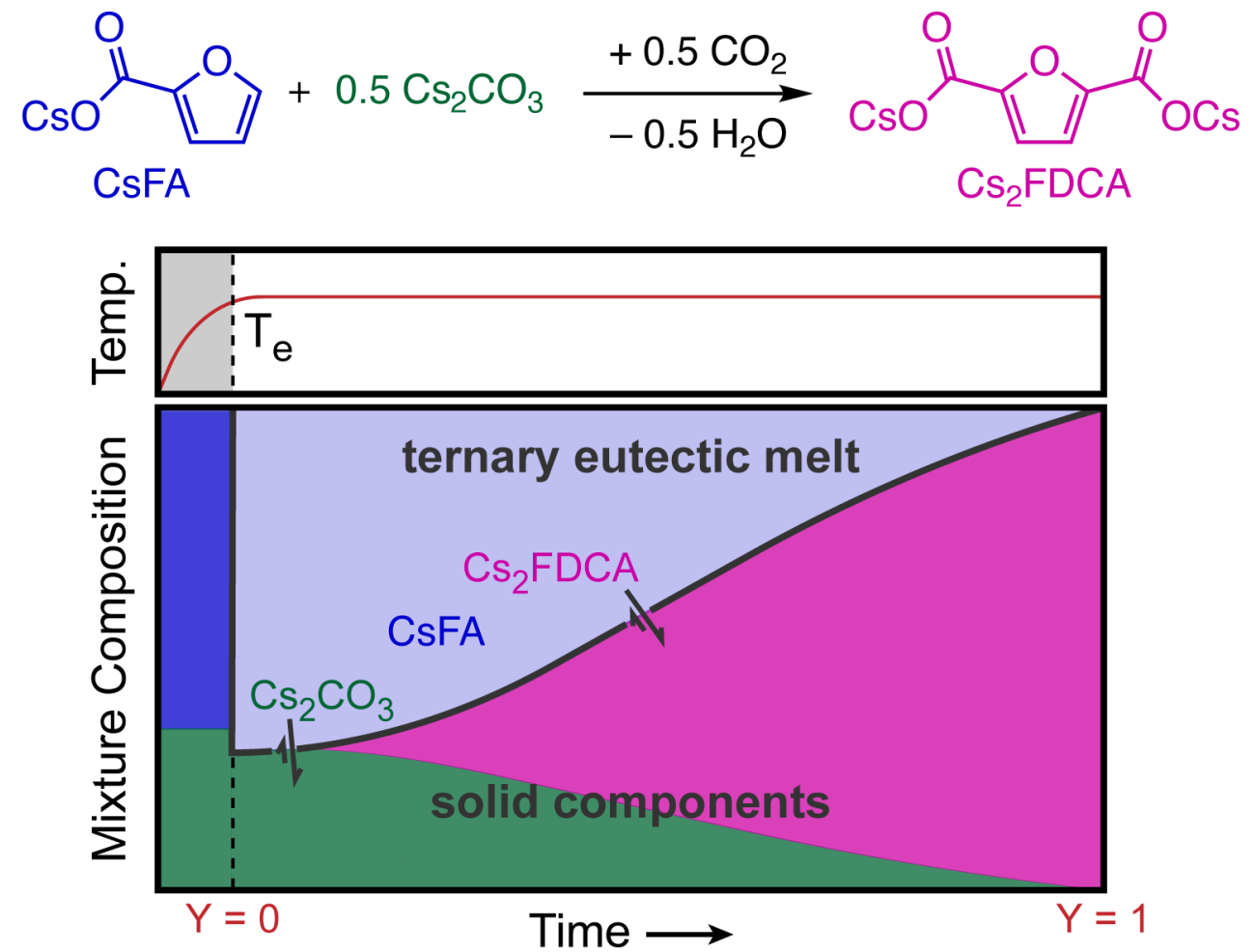

Figure 5 | Evolution of the reaction mixture during solvent-free carboxylation of cesium furoate.

two solid phases has been reported previously, ${ }^{26-29}$ although in these cases mechanical energy (e.g. ball milling) was needed to observe reactivity.

The higher melting point of KFA makes it infeasible to perform carboxylation with only KFA and $\mathrm{K}_{2} \mathrm{CO}_{3}$ because a eutectic cannot be accessed before decomposition. However, we have shown previously that adding a stoichiometric quantity of potassium isobutyrate to the $\mathrm{KFA}$ and $\mathrm{K}_{2} \mathrm{CO}_{3}$ mixture enables carboxylation to occur. ${ }^{10}$ Additionally, carboxylation can be performed in high yield using salts composed of furoate and carbonate with a mixture of $\mathrm{K}^{+}$and $\mathrm{Cs}^{+}$cations, even when only a small proportion of $\mathrm{Cs}^{+}$salts is used. ${ }^{11}$ While characterizing the phase behavior of these more complicated systems is beyond the scope of this study, the additional degrees of freedom created by using two cations or introducing another anion is expected to give rise to a relatively low-melting eutectic that enables the reaction to proceed. ${ }^{24,25}$ We note that the fraction of the salt mixture that exists in a molten state may be small enough such that there is no visible bulk melting event during a reaction. Regardless of composition, however, furoate carboxylation results in sintering of the salt particles, which is consistent with the formation of molten components during the reaction. 
Finally, the results above illuminate the factors that must be addressed in scaling furoate carboxylation. When the carboxylation is performed under quiescent conditions, the evolution of the phase components over time creates mass transport limitations. Specifically, as the ternary eutectic and solid $\mathrm{Cs}_{2} \mathrm{CO}_{3}$ phases decrease and the solid $\mathrm{Cs}_{2} \mathrm{FDCA}$ product accumulates, mass transport of $\mathrm{CO}_{2}$ and carbonate into the molten phase are expected to become increasingly difficult. Reactor design must therefore take into account the variation in mixture heterogeneity as a function of conversion and maintain a high effective contact area between the molten phase, the $\mathrm{CO}_{2}$ gas, and the $\mathrm{Cs}_{2} \mathrm{CO}_{3}$ phase. Reactors that continuously mix or grind the reaction components may be especially useful in this regard. ${ }^{30,31}$

The combination of PXRD and thermal analysis techniques has provided a detailed description of the phase composition of solvent-free furoate carboxylation. The results demonstrate that the reaction occurs in a molten phase dominated by $\mathrm{CsFA}$ with smaller amounts of the $\mathrm{Cs}_{2} \mathrm{CO}_{3}$ reactant and $\mathrm{Cs}_{2} \mathrm{FDCA}$ product. The diminution of the molten component and solid $\mathrm{Cs}_{2} \mathrm{CO}_{3}$ component as the reaction forms solid $\mathrm{Cs}_{2} \mathrm{FDCA}$ presents challenges for maintaining efficient mass transport which need to be managed to achieve optimal yields on large scale. The strategy for analyzing furoate carboxylation described here can be applied to other solvent-free systems to help identify compositional and operational changes that improve performance.

\section{Acknowledgements}

This work was supported by the U.S. Department of Energy (DOE) Advanced Research Projects Agency - Energy (ARPA-E) (DE-AR0000961). A.D.F. acknowledges support from a NASA Space Technology Research Fellowship. Powder X-ray diffraction was performed at the Advanced Photon Source, a DOE Office of Science User Facility operated for the DOE Office of Science by Argonne National Laboratory under Contract No. DE-AC02-06CH11357. Thermal analysis was

performed at the Stanford Nano Shared Facilities (SNSF), supported by the National Science Foundation under award ECCS-1542152. We thank Jana Maclaren and Andrey Yakovenko for experimental assistance, and Michael Toney and members of the Toney Laboratory for guidance on PXRD structure refinement. 


\section{References}

1. Loupy, A., Solvent-free Reactions. In Modern Solvents in Organic Synthesis, Knochel, P., Ed. Springer Berlin Heidelberg: Berlin, Heidelberg, 1999; pp 153-207.

2. Tanaka, K.; Toda, F., Solvent-Free Organic Synthesis. Chem. Rev. 2000, 100 (3), 1025-1074.

3. Garay, A. L.; Pichon, A.; James, S. L., Solvent-free synthesis of metal complexes. Chem. Soc. Rev. 2007, 36 (6), 846-855.

4. Bala, M. D.; Coville, N. J., Organometallic chemistry in the melt phase. J. Organomet. Chem. 2007, 692 (4), 709-730.

5. Martins, M. A. P.; Frizzo, C. P.; Moreira, D. N.; Buriol, L.; Machado, P., Solvent-free heterocyclic synthesis. Chem. Rev. 2009, 109 (9), 4140-4182.

6. Sarkar, A.; Santra, S.; Kundu, S. K.; Hajra, A.; Zyryanov, G. V.; Chupakhin, O. N.; Charushin, V. N.; Majee, A., A decade update on solvent and catalyst-free neat organic reactions: a step forward towards sustainability. Green Chem. 2016, 18 (16), 4475-4525.

7. Cintas, P.; Tabasso, S.; Veselov, V. V.; Cravotto, G., Alternative reaction conditions: Enabling technologies in solvent-free protocols. Curr. Opin. Green Sust. Chem. 2020, 21, 44-49.

8. Kulla, H.; Haferkamp, S.; Akhmetova, I.; Röllig, M.; Maierhofer, C.; Rademann, K.; Emmerling, F., In Situ Investigations of Mechanochemical One-Pot Syntheses. Angew. Chem. Int. Ed. 2018, 57 (20), 5930-5933.

9. Julien, P. A.; Germann, L. S.; Titi, H. M.; Etter, M.; Dinnebier, R. E.; Sharma, L.; Baltrusaitis, J.; Friščić, T., In situ monitoring of mechanochemical synthesis of calcium urea phosphate fertilizer cocrystal reveals highly effective water-based autocatalysis. Chem. Sci. 2020, 11 (9), 2350-2355.

10. Banerjee, A.; Dick, G. R.; Yoshino, T.; Kanan, M. W., Carbon dioxide utilization via carbonate-promoted C-H carboxylation. Nature 2016, 531 (7593), 215-9.

11. Dick, G. R.; Frankhouser, A. D.; Banerjee, A.; Kanan, M. W., A Scalable Carboxylation Route to 2,5-Furandicarboxylic Acid. Green Chem. 2017, 19, 2966-2972.

12. Banerjee, A.; Kanan, M. W., Carbonate-Promoted $\mathrm{CO}_{2}$ Hydrogenation to Multi-Carbon Oxygenates. ACS Cent. Sci. 2018, 4, 606-613.

13. Kanan, M. W.; Banerjee, A. Carbonate-promoted carboxylation reactions for the synthesis of valuable organic compounds. U.S. Patent 10,160,740, 2018.

14. Vechorkin, O.; Hirt, N.; Hu, X., Carbon dioxide as the $\mathrm{C} 1$ source for direct C-H functionalization of aromatic heterocycles. Org Lett 2010, 12 (15), 3567-9.

15. de Jong, E.; Dam, M.; Sipos, L.; Gruter, G., Furandicarboxylic acid (FDCA), a versatile building block for a very interesting class of polyesters. Biobased monomers, polymers, and materials 2012, 1105, 1-13.

16. Jensen, M. H.; Riisager, A., Chapter 5 - Advances in the synthesis and application of 2,5furandicarboxylic acid. In Biomass, Biofuels, Biochemicals, Saravanamurugan, S.; Pandey, A.; Li, H.; Riisager, A., Eds. Elsevier: 2020; pp 135-170.

17. Sajid, M.; Zhao, X.; Liu, D., Production of 2, 5-furandicarboxylic acid (FDCA) from 5hydroxymethylfurfural (HMF): recent progress focusing on the chemical-catalytic routes. Green Chem. 2018, 20 (24), 5427-5453.

18. Lide, D. R., CRC handbook of chemistry and physics. CRC press: 2004.

19. Butterhof, C.; Martin, T.; Milius, W.; Breu, J., Microphase separation with small amphiphilic molecules: crystal structure of preservatives sodium benzoate (E211) and potassium benzoate (E212). Z. Anorg. Allg. Chem. 2013, 639 (15), 2816-2821. 
20. Coelho, A. A., Whole-profile structure solution from powder diffraction data using simulated annealing. J. Appl. Crystallogr. 2000, 33 (3), 899-908.

21. Hatibarua, J.; Parry, G. S., A crystallographic study of the acetates of potassium, rubidium and caesium. Acta Crystallogr. B Struct. Sci. 1972, 28 (10), 3099-3100.

22. Van Deun, R.; Ramaekers, J.; Nockemann, P.; Van Hecke, K.; Van Meervelt, L.; Binnemans, K., Alkali-Metal Salts of Aromatic Carboxylic Acids: Liquid Crystals without Flexible Chains. Eur. J. Inorg, Chem. 2005, 2005 (3), 563-571.

23. Nelson, P. N.; Taylor, R. A., Theories and experimental investigations of the structural and thermotropic mesomorphic phase behaviors of metal carboxylates. Appl. Petrochem. Res. 2014, 4 (3), 253-285.

24. Rothenberg, G.; Downie, A. P.; Raston, C. L.; Scott, J. L., Understanding solid/solid organic reactions. J. Am. Chem. Soc. 2001, 123 (36), 8701-8708.

25. Daněk, V., Chapter 3 - Phase Equilibria. In Physico-Chemical Analysis of Molten Electrolytes, Daněk, V., Ed. Elsevier Science: Amsterdam, 2006; pp 107-219.

26. Braga, D.; Grepioni, F., Reactions between or within molecular crystals. Angew. Chem. Int. Ed. 2004, 43 (31), 4002-4011.

27. Balema, V. P.; Wiench, J. W.; Pruski, M.; Pecharsky, V. K., Mechanically induced solid-state generation of phosphorus ylides and the solvent-free wittig reaction. J. Am. Chem. Soc. 2002, 124 (22), 6244-6245.

28. Balema, V. P.; Wiench, J. W.; Pruski, M.; Pecharsky, V. K., Solvent-free mechanochemical synthesis of two $\mathrm{Pt}$ complexes: cis- $\left(\mathrm{Ph}_{3} \mathrm{P}\right)_{2} \mathrm{PtCl}_{2}$ and cis- $\left(\mathrm{Ph}_{3} \mathrm{P}\right)_{2} \mathrm{PtCO}_{3}$. Chem. Comm. 2002, (15), 1606-1607.

29. Dolotko, O.; Wiench, J. W.; Dennis, K. W.; Pecharsky, V. K.; Balema, V. P., Mechanically induced reactions in organic solids: liquid eutectics or solid-state processes? New J. Chem. 2010, $34(1), 25-28$.

30. Crawford, D. E.; Miskimmin, C. K. G.; Albadarin, A. B.; Walker, G.; James, S. L., Organic synthesis by Twin Screw Extrusion (TSE): continuous, scalable and solvent-free. Green Chem. 2017, 19 (6), 1507-1518.

31. Rodriguez, B.; Bruckmann, A.; Rantanen, T.; Bolm, C., Solvent-free carbon-carbon bond formations in ball mills. Adv. Synth. Catal. 2007, 349 (14-15), 2213-2233. 